PROCEEDINGS OF THE

AMERICAN MATHEMATICAL SOCIETY

Volume 135, Number 1, January 2007, Pages 5-11

S 0002-9939(06)08565-0

Article electronically published on August 16, 2006

\title{
ON BREDON HOMOLOGY OF ELEMENTARY AMENABLE GROUPS
}

\author{
RAMÓN J. FLORES AND BRITA E. A. NUCINKIS
}

(Communicated by Jonathan I. Hall)

\begin{abstract}
We show that for elementary amenable groups the Hirsch length is equal to the Bredon homological dimension. This also implies that countable elementary amenable groups admit a finite-dimensional model for $\underline{E} G$ of dimension less than or equal to the Hirsch length plus one. Some remarks on groups of type $\mathrm{FP}_{\infty}$ are also made.
\end{abstract}

Bredon cohomology groups have been defined first for finite groups [4, but this definition can easily be extended to infinite groups (see [7, 17, 18, 20, 23, 24]) and has received some attention through its connection with classifying spaces for proper actions [3, 16, 18, 22. Bredon homology can be defined in a straightforward way and plays an important role in connection with the Baum-Connes conjecture 22 .

Motivated by Stammbach's result 25] that for soluble groups the Hirsch length is equal to the homological dimension over $\mathbb{Q}$, it was shown by the second author 24 that for nilpotent groups the Bredon homological dimension is equal to the Hirsch length. In his survey Lück [19] gave an account of the well-known result that polycylic-by-finite groups admit a model for $\underline{E} G$ of dimension equal to the Hirsch length, which also implies that Hirsch length and Bredon homological dimension are equal. Here we show that these results on the Bredon homological dimension can be extended to the class of elementary amenable groups, a class which contains all soluble-by-finite groups.

Denote by $\mathfrak{X}$ the class of elementary amenable groups. This is the smallest class of groups, which contains all abelian and all finite groups and is closed under extensions and directed unions. A more constructive description of this class is due to Kropholler-Linnell-Moody [15], which we recall here since it is used in the proof of our main theorem:

Let $\mathfrak{X}$ and $\mathfrak{Y}$ denote classes of groups. We say a group belongs to a class $\mathfrak{X Y Y}$ if it is an extension of a group belonging to $\mathfrak{X}$ by a group belonging to $\mathfrak{Y}$. Also, a group belongs to $L \mathfrak{X}$ if each finitely generated subgroup belongs to $\mathfrak{X}$.

\footnotetext{
Received by the editors July 20, 2005.

2000 Mathematics Subject Classification. Primary 20J05, 18G20.

Key words and phrases. Elementary amenable group, Bredon homology.

This work was partially supported by MCYT grant BFM2001-2035.
} 
From now on $\mathfrak{Y}$ denotes the class of all finitely generated abelian-by-finite groups. For each ordinal $\alpha$ we define the class $\mathfrak{X}_{\alpha}$ inductively

$$
\begin{aligned}
& \mathfrak{X}_{0}=\{1\}, \\
& \mathfrak{X}_{\alpha}=\left(L \mathfrak{X}_{\alpha-1}\right) \mathfrak{Y} \quad \text { if } \alpha \text { is a successor ordinal, } \\
& \mathfrak{X}_{\alpha}=\bigcup_{\beta<\alpha} \mathfrak{X}_{\beta} \quad \text { if } \alpha \text { is a limit ordinal. }
\end{aligned}
$$

The class of all elementary amenable groups is now defined by setting

$$
\mathfrak{X}=\bigcup_{\alpha \geq 0} \mathfrak{X}_{\alpha} .
$$

Hillman [11 has extended the notion of Hirsch length of a soluble group to the class of elementary amenable groups as follows: Let $\alpha$ be the least ordinal such that $G$ belongs to $\mathfrak{X}_{\alpha}$. If $\alpha=1$, then $G$ is finitely generated abelian-by-finite and the Hirsch length $h G$ is equal to the torsion-free rank of the abelian normal subgroup of $G$ of finite index. For $\alpha>1$, there is a normal subgroup $N$ of $G$, which lies in some $L \mathfrak{X}_{\beta}$, and the Hirsch length of all groups belonging to $\mathfrak{X}_{\beta}$ has been defined. The Hirsch length of $N$ is now the least upper bound of the Hirsch lengths of the $\mathfrak{X}_{\beta}$-subgroups of $N$. The quotient $G / N$ now lies in $\mathfrak{Y}$, and the Hirsch length of $G$ is defined by

$$
h G=h N+h(G / N) .
$$

In [12] (see also 26] for an alternative proof) it was shown that elementary amenable groups of finite Hirsch length are in fact locally-finite-by-virtually-soluble, a fact which is not used in the proof of our theorem.

Theorem 1. Let $G$ be an elementary amenable group of finite Hirsch length $n$. Then $\underline{\mathrm{hd}} G=n$.

Let us begin with collecting the necessary definitions and facts about Bredon homology. Basics can be found in [17, 7, 22, See also 20] for spectral sequences and [19, 24] for facts about Bredon (co)homological dimension.

Let $G$ be an arbitrary group and $\mathfrak{F}$ a family of subgroups closed under subgroups and conjugation. For this note we are only concerned with the family $\mathfrak{F}$ of all finite subgroups of $G$. Consider the orbit category $\mathcal{O}_{\mathfrak{F}} G$, which is a category with objects the cosets $G / H$, where $H \in \mathfrak{F}$ and the morphisms are $G$-maps $G / H \rightarrow G / K$, where $G / H$ and $G / K$ are objects in $\mathcal{O}_{\mathfrak{F}} G$. The two functor categories of interest from $\mathcal{O}_{\mathfrak{F}} G$ to $\mathfrak{A} \mathfrak{b}$, the category of abelian groups, are $\operatorname{Mod}_{\mathfrak{F}}-G$, the category of contravariant functors $M(-): \mathcal{O}_{\mathfrak{F}} G \rightarrow \mathfrak{A} \mathfrak{b}$, also called right $\mathcal{O}_{\mathfrak{F}} G$-modules, and $G-\operatorname{Mod}_{\mathfrak{F}}$, the category of covariant functors $N(-): \mathcal{O}_{\mathfrak{F}} G \rightarrow \mathfrak{A} \mathfrak{b}$, also called left $\mathcal{O}_{\mathfrak{F}} G$-modules.

These are abelian categories, and we have the notion of kernels, cokernels, arbitrary coproducts and short exact sequences. We can also define chain complexes, exact sequences, resolutions and chain homotopies in the usual manner.

The category $\operatorname{Mod}_{\mathfrak{F}}-G$ also has enough projectives, and hence every right $\mathcal{O}_{\mathfrak{F}} G$ module $M$ admits a projective resolution, denoted $P_{*} \rightarrow M$, which is used to define the derived functors $\operatorname{Ext}_{\mathfrak{F}}^{*}(M, N)$ :

$$
\operatorname{Ext}_{\mathfrak{F}}^{*}(M, N)=H^{*}\left(\operatorname{mor}\left(P_{*}, N\right)\right) \text {. }
$$

We now define the categorical tensor product to be the abelian group

$$
M(-) \otimes_{\mathfrak{F}} N(-)=\bigoplus_{K \in \mathfrak{F}} M(G / K) \otimes N(G / K) / \sim,
$$


where $\sim$ is the equivalence relation generated by $\varphi^{*}(m) \otimes n=m \otimes \varphi_{*}(n)$ with $m \in M(G / L)$ and $n \in N(G / K)$, and $\varphi: G / K \rightarrow G / H$ is a $G$-map.

Let $\operatorname{Tor}_{n}^{\mathfrak{F}}(-, N)$ denote the $n$-th left derived functor of the tensor product $-\otimes_{\mathfrak{F}} N$. We say a right $\mathcal{O}_{\mathfrak{F}} G$-module $M$ is flat if the functor $M \otimes_{\mathfrak{F}}-$ is exact. The Yoneda isomorphism implies that projective $\mathcal{O}_{\mathfrak{F}} G$-modules are flat.

Analogous to ordinary homology and cohomology we define (co)homological dimensions via resolutions of the constant functor $\underline{\mathbb{Z}}$. We define $\underline{\mathrm{cd}} G$ to be the shortest length of a projective resolution of $\underline{\mathbb{Z}}$ and $\underline{\mathrm{hd}} G$ to be the shortest length of a flat resolution of $\underline{\mathbb{Z}}$.

There now follow some facts about these quantities which we shall use later. For details the reader is referred to [24, Section 4]. Let $G$ be an arbitrary group. Then

$$
\underline{\mathrm{hd}} G \leq \underline{\mathrm{cd}} G .
$$

If, in addition, $G$ is countable, then

$$
\underline{\mathrm{cd}} G \leq \underline{\mathrm{hd}} G+1 \text {. }
$$

Following 20 we say a family $\mathfrak{F}$ is bounded if each $K \in \mathfrak{F}$ is contained in a maximal $M \in \mathfrak{F}$. Let $\left\{G_{\lambda}\right\}_{\lambda \in \Lambda}$ be a direct system of groups. Then, for the direct limit $G=\lim _{\rightarrow} G_{\lambda}$ and $\mathfrak{F}$ a bounded family of finite subgroups of $G$,

$$
\underline{\mathrm{hd}} G=\max \left\{\underline{\mathrm{hd}} G_{\lambda}\right\} .
$$

Usually, the family of all finite subgroups $\mathfrak{F}$ is not bounded. However one can define a bounded family $\hat{\mathfrak{F}}$, the completion of $\mathfrak{F}$, as follows:

$$
\hat{\mathfrak{F}}=\mathfrak{F} \cup\left\{\operatorname{colim} L_{i} ; L_{1} \leq L_{2} \leq \ldots \text { a chain of subgroups } L_{i} \in \mathfrak{F}\right\} \text {. }
$$

It now follows [20, Theorem 4.8] that

$$
\underline{\mathrm{hd}} G:=\operatorname{hd}_{\mathfrak{F}} G \leq \operatorname{hd}_{\hat{\mathfrak{F}}} G .
$$

Lemma 2. Let $\mathfrak{X}$ be a class of elementary amenable groups such that for all $H$ belonging to $\mathfrak{X F}, \operatorname{hd}_{\mathfrak{F}} H=h H$, then for all $G \in(L \mathfrak{X}) \mathfrak{F}$ we also have

$$
\operatorname{hd}_{\widehat{\mathfrak{F}}} G=h G .
$$

Proof. Let $G \in(L \mathfrak{X}) \mathfrak{F}$. We therefore have a group extension

$$
G_{0} \hookrightarrow G \rightarrow Q,
$$

where $G_{0} \in L \mathfrak{X}$ and $Q$ is finite. Now $G=\lim G_{\lambda}$, where $G_{\lambda}$ are the finitely generated subgroups of $G$. Hence, for each $\lambda, H_{\lambda}=\overrightarrow{G_{0}} \cap G_{\lambda}$ is finitely generated and hence belongs to $\mathfrak{X}$, and $G_{\lambda}$ belongs to $\mathfrak{X F}$. By the assumption, $\operatorname{hd}_{\hat{\mathfrak{F}}} G_{\lambda}=h G_{\lambda}$. Also, $\hat{\mathfrak{F}}$ is bounded in the sense of 20. Hence, by (3) we have the following equalities:

$$
\operatorname{hd}_{\hat{\mathfrak{F}}} G=\operatorname{hd}_{\hat{\mathfrak{F}}}\left(\lim _{\rightarrow} G_{\lambda}\right)=\max \left\{\operatorname{hd}_{\widehat{\mathcal{F}}} G_{\lambda}\right\}=\max \left\{h G_{\lambda}\right\}=h G .
$$

We also need to know the behaviour of Bredon homological dimension under group extensions $N \hookrightarrow G \rightarrow Q$. Let $\mathfrak{H}=\{S \leq G: N \leq S$ and $S / N \in \mathfrak{F}(Q)\}$. Assume that there is an integer $n$ such that for every $S \in \mathfrak{H}, \underline{h d} S \leq n$. Then an application of Martinez' spectral sequence [20, 5.1] yields:

$$
\underline{\mathrm{hd}} G \leq n+\underline{\mathrm{hd}} Q \text {. }
$$


The conditions of (4) are obviously satisfied when $Q$ is torsion-free. Nevertheless we cannot hope to get a result like (4) for arbitrary group extensions. Ian Leary and the second author give examples of virtually torsion-free groups $G$, where $\underline{\mathrm{cd}} G$ is strictly greater than $\mathrm{cd} H$ for $H$ a torsion-free subgroup of finite index; see [16. Example 12]. Analogously one sees 24] that for these groups we also have $\underline{\mathrm{cd}} G=\underline{\mathrm{hd}} G>h d G=c d H$.

Example 3. It is a well-known fact (see for example [19, Example 5.25]) that for a polycyclic-by-finite group $G$ there is always a model for an $\underline{E} G$ of dimension equal to the Hirsch-rank of its polycyclic subgroups of finite index. Hence, $\underline{\mathrm{cd}} G=\underline{\mathrm{hd}} G=$ $h G$.

Polycyclic groups have a bound on the orders of the finite subgroups, and hence $\mathfrak{F}=\hat{\mathfrak{F}}$, which means that $\mathrm{hd}_{\hat{\mathfrak{F}}} G=\underline{\mathrm{hd}} G=h G$.

One can also show [24,6.1] that for a nilpotent group $G$,

$$
\underline{\mathrm{hd}} G=h G \text {. }
$$

Proof of Theorem 1. By the above, the statement and also the assumptions of Lemma 2 are obviously true for groups belonging to $\mathfrak{X}_{1}$. Now, let $G$ be an arbitrary group belonging to $\mathfrak{X}$ and let $\alpha$ be the least ordinal such that $G \in \mathfrak{X}_{\alpha}$. Assume that $\alpha$ is a successor ordinal. Therefore $G$ has a normal subgroup $N \in L \mathfrak{X}_{\alpha-1}$, and there is an extension

$$
N \hookrightarrow G \rightarrow Q
$$

with $Q$ belonging to $\mathfrak{Y}$. By transfinite induction, each group lying in $\mathfrak{X}_{\alpha-1}$ satisfies the assumptions of Lemma 2, and hence for each finite extension $N^{\prime}$ of $N$ we have

$$
\operatorname{hd}_{\hat{\mathfrak{F}}} N^{\prime}=h N^{\prime}=h N \text {. }
$$

Hence we can apply the spectral sequence [20, 5.2] to obtain

$$
\operatorname{hd}_{\hat{\mathfrak{F}}} G \leq \operatorname{hd}_{\widehat{\mathfrak{F}}} N+\operatorname{hd}_{\hat{\mathfrak{F}}} Q=h N+h Q=h G .
$$

For $\alpha$ a limit ordinal an application of [24, 4.2(ii)] also yields

$$
\operatorname{hd}_{\widehat{\mathfrak{F}}} G=h G .
$$

[24, Proposition 4.3] and an analogue to [11, Lemma 2] imply

$$
\underline{\mathrm{hd}} G \geq \operatorname{hd}_{\mathbb{Q}} G \geq h G .
$$

An application of [20,5.2] as mentioned above yields

$$
\operatorname{hd}_{\hat{\mathfrak{F}}} G \geq \underline{\operatorname{hd}} G \geq h G,
$$

thus proving the theorem.

There now follows a remark on the dimension of the classifying space for proper actions, $\underline{E} G$. It is well known that the minimal dimension of a model for $\underline{E} G$, called $\underline{\operatorname{gd} G}$, is equal to $\underline{\mathrm{cd}} G$ provided that $\underline{\mathrm{cd}} G \neq 2$; see [17] and [3] for an example of a group with $\underline{\mathrm{cd}} G=2$ yet $\operatorname{gd} G=3$. In $[21$ it was proved that soluble groups of finite Hirsch length admit a finite-dimensional model for $\underline{E} G$, although no bound on this dimension was given. Hence, by Serre's Theorem [5], every soluble-by-finite group admits a finite-dimensional model for $\underline{E} G$. As mentioned earlier, every elementary amenable group of finite Hirsch length is actually locally-finite-by-virtually-soluble 12, 26. Locally finite groups of cardinality $\aleph_{n}$ admit an $(n+1)$-dimensional model for $\underline{E} G$; see [6, 8]. Hence an application of Martinez' spectral sequence (see [20, 
5.1]) yields that elementary amenable groups of finite Hirsch length admit a finitedimensional model for $\underline{E} G$. We recover this fact for countable elementary amenable groups by applying Theorem 1 and are also able to provide a bound on the minimal dimension of a model for $\underline{E} G$. The only additional ingredient is (2).

Corollary 4. Let $G$ be a countable elementary amenable group of finite Hirsch length $h G$. If $\underline{\operatorname{cd}} G \neq 2$, then $G$ admits a model for $\underline{E} G$ of dimension less than or equal to $h G+1$.

Groups of type $\mathbf{F P}_{\infty}$. A group $G$ is said to be of type $\mathrm{FP}_{\infty}$ if the trivial module $\mathbb{Z}$ admits a resolution with finitely generated projective $\mathbb{Z} G$-modules. Analogously, a group $G$ is of Bredon type $\mathrm{FP}_{\infty}$, or $\underline{\mathrm{FP}}_{\infty}$ for short, if the constant Bredon functor $\underline{\mathbb{Z}}$ admits a resolution

$$
\underline{P}_{*} \rightarrow \underline{\mathbb{Z}}
$$

with finitely generated projective Bredon modules $\underline{P}_{n}$, for all $n \geq 0$. A precise definition of a finite generation of Bredon modules can be found in [24, §2]. Analogously to the torsion-free case [2, 4.6(c)], an application of [24, 3.3, 4.1] leads to the following result.

Proposition 5. Let $G$ be a group of type $\underline{\mathrm{FP}}_{\infty}$. Then

$$
\underline{\mathrm{hd}} G=\underline{\mathrm{cd}} G .
$$

For a torsion-free soluble group the conjecture that $\operatorname{cd} G=\operatorname{hd} G=h G$ is equivalent to the group being of type $\mathrm{FP}_{\infty}$ has been open for quite some time with partial results in [1, 9, 10, and was finally proven by Kropholler [13]. Hence we are led to conjecture the following analogue.

Conjecture 1. Let $G$ be an elementary amenable group. Then the following are equivalent:

(i) $\underline{\mathrm{cd}} G=\underline{\mathrm{hd}} G=h G$,

(ii) $G$ is of type $\underline{\mathrm{FP}}_{\infty}$.

We do not know how to prove (i) $\Rightarrow$ (ii), but there now follow some indications as to why it might be correct.

Lemma 6. Let $G$ be an elementary amenable group such that $\underline{\mathrm{cd}} G=h G$; then $\operatorname{cd}_{\mathbb{Q}} G=h G$.

Proof. $h G \leq \operatorname{cd}_{\mathbb{Q}} G \leq \underline{\mathrm{cd}} G=h G$, where the first inequality follows from [11, Lemma 2].

From now on let $G$ be an elementary amenable group of finite Hirsch length. If $G$ has a bound on the orders of its finite subgroups, we can reduce to the case when $G$ is virtually torsion-free soluble. As before, [26] implies the existence of an extension

$$
T \hookrightarrow G \rightarrow Q
$$

with $T$ locally finite and hence finite, and $Q$ virtually torsion-free soluble. [24, 5.5] now implies that $\underline{\mathrm{cd}} G=\underline{\mathrm{cd}} Q$ and $\underline{\mathrm{hd}} G=\underline{\mathrm{hd}} Q$.

Thus we obtain the following partial result.

Proposition 7. Let $G$ be an elementary amenable group with $\underline{\mathrm{cd}} G=h G$, which has a bound on the orders of its finite subgroups. Then $G$ is of type $\mathrm{FP}_{\infty}$. 
Proof. By the above remark we may assume that $G$ has a torsion-free soluble subgroup $H$ of finite index. Suppose $G$ is not of type $\mathrm{FP}_{\infty}$. This implies that $H$ is not type $\mathrm{FP}_{\infty}$ either, and hence by the aforementioned result of Kropholler 13, $\operatorname{cd}_{\mathbb{Q}} H \neq \operatorname{hd}_{\mathbb{Q}} H$. Hence,

contradicting Lemma 6 .

$$
c d_{\mathbb{Q}} G \neq \mathrm{hd}_{\mathbb{Q}} G,
$$

Thus the following conjecture would imply Conjecture 1 in the above case.

Conjecture 2. Let $G$ be a group of type $\mathrm{FP}_{\infty}$ containing a torsion-free soluble group of finite index. Then $G$ is of type $\underline{\mathrm{FP}}_{\infty}$.

Since groups of type $\mathrm{FP}_{\infty}$ with finite cohomological dimension over $\mathbb{Q}$ have a bound on the orders of their finite subgroups 14, a positive answer of Conjecture 2 would imply one direction of the following final conjecture.

Conjecture 3. Let $G$ be an elementary amenable group of finite Hirsch length. Then $\underline{\mathrm{cd}} G=h G$ is equivalent to $G$ being of type $\mathrm{FP}_{\infty}$.

\section{ACKNOWLEDGEMENTS}

The authors thank the Forschungsinstitut für Mathematik, ETH Zürich and the Departamento de Matemáticas, Universidad Autónoma de Barcelona for their hospitality during their visits in April 2004 and June 2005, respectively.

\section{REFERENCES}

[1] B. Baumslag and R. Bieri, Constructable solvable groups, Math. Z. 151 (1976), 249-257. MR0422422 (54:10411)

[2] R. Bieri, Homological dimension of discrete groups, Queen Mary College Mathematics Notes, London (1976). MR0466344 (57:6224)

[3] N.P. Brady, I.J. Leary and B.E.A. Nucinkis, On algebraic and geometric dimensions for groups with torsion, J. London Math. Soc. 64 (2) (2001), 489-500. MR1853466 (2002h:57007)

[4] G.E. Bredon, Equivariant cohomology theories, Springer Lecture Notes in Math. 34 (1967). MR0214062 (35:4914)

[5] K.S. Brown, Cohomology of groups, Graduate Texts in Mathematics 87, Springer-Verlag (1982). MR0672956 (83k:20002)

[6] W. Dicks, I.J. Leary, P.H. Kropholler and S. Thomas, Classifying spaces for proper actions of locally finite groups, J. Group Theory 5 (4) (2002), 453-480. MR.1931370 (2003g:20064)

[7] T. tom Dieck, Transformation Groups, de Gruyter Studies in Mathematics 8 (1987). MR0889050 (89c:57048)

[8] M.J. Dunwoody, Accessibility and groups of cohomological dimension 1, Proc. London Math. Soc. 38 (1997), 193-215. MR0531159 (80i:20024)

[9] D. Gildenhuys and R. Strebel, On the cohomological dimension of soluble groups, Cand. Math. Bull. 24 (4), (1981), 385-392. MR.0644526 (83h:20047)

[10] D. Gildenhuys and R. Strebel, On the cohomological dimension of soluble groups II, J. Pure Appl. Algebra 26 (1982), 293-323. MR0678526 (84c:20059)

[11] J.A. Hillman, Elementary amenable groups and 4-manifolds with Euler characteristic 0, J. Austral. Math. Soc Ser. A 50 (1) (1991), 160-170. MR1094067 (92g:20057)

[12] J.A. Hillman and P.A. Linnell, Elementary amenable groups of finite Hirsch length are locallyfinite by virtually solvable, J. Austral. Math. Soc Ser. A 52 (2) (1992), 237-241. MR1143191 (93b:20067)

[13] P.H. Kropholler, Cohomological dimension of soluble groups, J. Pure Appl. Algebra 43 (1986), 281-287. MR 0868988 (88h:20063)

[14] P.H. Kropholler, On groups of type $F P_{\infty}$, J. Pure Appl. Algebra 90 (1) (1993), 55-67. MR:1246274 (94j:20051b) 
[15] P.H. Kropholler, P.A. Linnell and J.A. Moody, Applications of a new $K$-theoretic theorem to soluble groups rings, Proc. Amer. Math. Soc. 104 (3) (1988), 675-684. MR0964842 (89j:16016)

[16] I.J. Leary and B.E.A. Nucinkis, Some groups of type VF, Invent. Math. 151 (1) (2003), 135-165. MR1943744 (2003k:20086)

[17] W. Lück, Transformation groups and algebraic K-theory, Lecture Notes in Mathematics 1408 Springer, Berlin (1989). MR1027600 (91g:57036)

[18] W. Lück, The type of the classifying space for a family of subgroups, J. Pure Appl. Algebra 149 (2000), 177-203. MR 1757730 (2001i:55018)

[19] W. Lück, Survey on Classifying Spaces for Families of Subgroups, Preprintreihe SFB 478 Geometrische Strukturen in der Mathematik 308 (2004).

[20] C. Martinez-Pérez, A spectral sequence in Bredon (co)homology, J. Pure Appl. Algebra 176 (2002), 161-173. MR1933713 (2003h:20095)

[21] G. Mislin, On the classifying space for proper actions, In cohomological Methods in Homotopy Theory, Progress in Mathematics 196, Birkhäuser (2001). MR.1851258(2002f:55032)

[22] G. Mislin, Equivariant K-homology of the classifying space for proper actions, In Notes on the Advances Course on proper group actions. CRM Barcelona (2001). MR2027169 (2005e:19008)

[23] B.E.A. Nucinkis, Is there an easy algebraic characterisation of universal proper $G$-spaces?, Manuscripta Math. 102 (2000), 335-345. MR.1777524 (2001h:20075)

[24] B.E.A. Nucinkis, On dimensions in Bredon homology, Homology, Homotopy Appl. 6 (1) (2004), 33-47. MR2061566 (2005c:20092)

[25] U. Stammbach, On the weak homological dimension of the group algebra of solvable groups, J. London Math. Soc. 2 (2) (1970), 567-570. MR0263927 (41:8526)

[26] B.A.F. Wehrfritz, On elementary amenable groups of finite Hirsch number, J. Austral. Math. Soc Ser. A 58 (2), (1995), 219-221. MR1323993 (96a:20050)

Departamento de Matemáticas, Universidad Autónoma de Barcelona, E 08193 BelLATERRA, SPAin

E-mail address: ramonj@mat.uab.es

Current address: Departamento de Estadística, Universidad Carlos III, Campus de Colmenarejo, 2228270 Colmenarejo (Madrid), Spain

E-mail address: rflores@est-econ.uc3m.es

School of Mathematics, University of Southampton, Southampton, SO 17 1BJ, United KINGDOM

E-mail address: B.E.A.Nucinkis@soton.ac.uk 